\title{
Evaluating Diabetic Foot Infection with Magnetic Resonance Imaging: Kuwait Experience
}

\author{
Hanaa A. Al-Khawari ${ }^{a}$ Osama M. Al-Saeed ${ }^{a}$ Taleb H. Jumaa ${ }^{b}$ Fayaz Chishti $^{b}$ \\ a Department of Radiology, Faculty of Medicine, Kuwait University, and \\ ${ }^{b}$ Departments of Surgery and Radiology, Al-Amiri Hospital, Kuwait
}

\section{Key Words}

Magnetic resonance imaging - Diabetic foot infection •

Diabetic foot osteomyelitis

\begin{abstract}
Objective: The aim of this study was to evaluate the capability of magnetic resonance imaging (MRI) to depict and characterize the changes seen in diabetic foot infections. Subjects and Methods: MRI studies of 29 diabetic patients with suspected foot infection were evaluated. Sagittal and transverse $T_{1}$-weighted images before and after intravenous gadolinium, and transverse fat-suppressed $\mathrm{T}_{2}$-weighted images were performed on the affected regions. The MRI findings were compared to subsequent clinical and/or histopathological findings. Results: The MRI findings were: osteomyelitis in 14 patients, abscess in 5, cellulitis in 26, tenosynovitis in 4 and neuropathic joint in 8 . Three cases were normal. Pathological confirmations were obtained in 19 patients. MRI and histological diagnosis were in concordance in $79 \%$ of osteomyelitis cases, $100 \%$ of neuropathy cases and $100 \%$ of cellulitis cases. The sensitivity and specificity of MRI in diagnosing osteomyelitis were 100 and $63 \%$, respectively. The positive predictive and negative predictive values, and the accuracy were 79,100 and $84 \%$, respectively. MRI helped surgical planning for limb salvage procedures in 6 of the osteomyelitis cases and in a cellulitis case. Conclusion: The results indicate that MRI is
\end{abstract}

a sensitive and accurate imaging modality for the evaluation of foot infections in diabetic patients and for planning proper treatment.

Copyright (C) 2005 S. Karger AG, Basel

\section{Introduction}

Diabetes mellitus is a common disorder among both children and adults with a total prevalence of $14.8 \%$ among Kuwaitis (5.7 and 18.3\% for age groups 20-39 and 40-59, respectively) [1]. Diabetic foot encompasses a group of abnormalities which results from ischemia, infection and sensory neuropathy, all of which may lead to tissue necrosis and necessitate amputation. In fact diabetes is the main cause of nontraumatic lower extremity amputation, which is 15-40 times more common in this group than in nondiabetics [2]. The diagnosis of diabetic foot osteomyelitis is usually a challenge to the clinician since it is difficult both clinically and radiologically to differentiate it from acute rapidly progressing neuroarthropathy. This distinction is essential for planning proper therapy and ensuring limb salvage, since osteomyelitis might require amputation in some cases while neuropathy requires conservative therapy only given that it has better prognosis [3].

Magnetic resonance imaging (MRI) has been shown to be useful in identifying musculoskeletal pathology, has high accuracy in diagnosing and revealing the extent of

\section{KARGER}

Fax +4161306 1234

E-Mail karger@karger.ch

www.karger.com
(C) 2005 S. Karger AG, Basel

1011-7571/05/0143-0165\$22.00/0

Accessible online at:

www.karger.com/mpp
Dr. Hanaa Al-Khawari

Kuwait University, Faculty of Medicine

Department of Radiology, PO Box 24923

13110 Safat (Kuwait)

Tel./Fax +965 5330473, E-Mail drhana@yahoo.com 

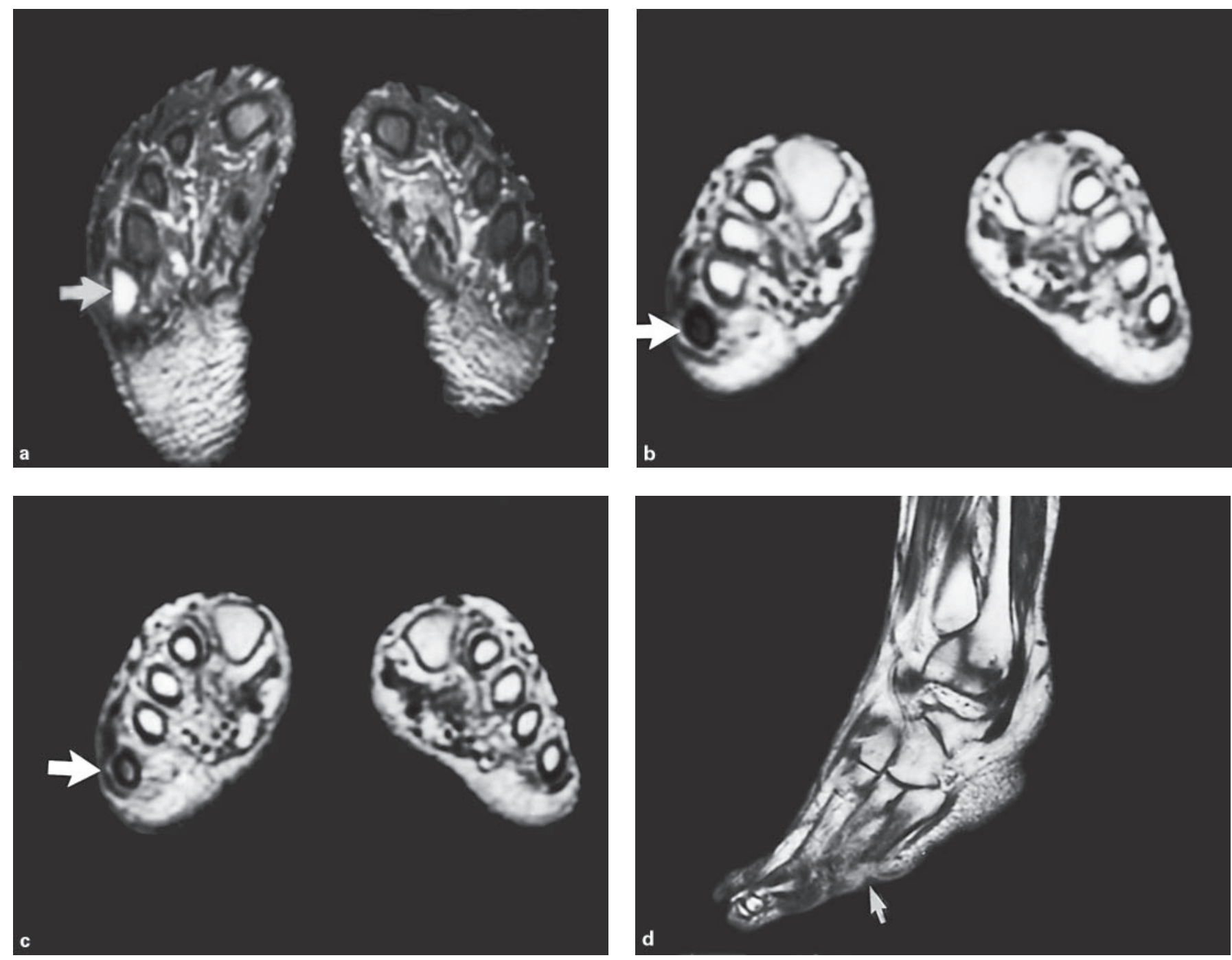

Fig. 1. Osteomyelitis. a Transverse $\mathrm{FST}_{2} \mathrm{WI}$ through the shafts of metatarsal bones shows increased signal intensity of the right 5 th metatarsal bone marrow (arrow). b Transverse $T_{1}$ WI shows low signal intensity of the bone marrow of the right 5 th metatarsal shaft (arrow). c Transverse postgadolinium $\mathrm{T}_{1}$ WI show focal bone marrow enhancement of the same region (arrow). d Sagittal postgadolinium $\mathrm{T}_{1}$ WI shows an ulcer close to the right 5 th metatarsal bone. Osteomyelitis was confirmed by subsequent bone biopsy.

musculoskeletal infections [4-8] and frequently dictates the course of treatment. We reviewed foot and ankle MRI images of 29 diabetic patients clinically suspected to have diabetic foot infection in an attempt to evaluate the capability of this technique to depict and characterize the changes present in osteomyelitis, soft tissue infections, deep seated abscesses, tenosynovitis, and neuropathic arthropathy, and to correlate the findings with clinical follow-up and/or histopathology.

\section{Subjects and Methods}

MRI studies of ankles and feet of 29 diabetic patients referred from the Diabetic Foot Clinic in Al-Amiri Hospital, Kuwait, from August 2000 to July 2002 clinically suspected of having ankle and/ or foot infection were evaluated. Seventeen were male and $12 \mathrm{fe}-$ male with an age range of 41-81 years (mean of 61).

MRI examinations were performed using a 1.0-tesla superconducting magnet (General Electric, Signa Horizon). Surface coil (head coil) was used in all cases. Sagittal and transverse spin echo $\mathrm{T}_{1}$-weighted images ( $\mathrm{T}_{1} \mathrm{WI}$ ) (TR $500 \mathrm{~ms}$, TE $20 \mathrm{~ms}$, NEX 2) before and after intravenous gadolinium $(0.1 \mathrm{ml} / \mathrm{kg})$ were obtained, and transverse fat suppressed spin-echo $\mathrm{T}_{2}$-weighted images $\left(\mathrm{FST}_{2} \mathrm{WI}\right)$ 

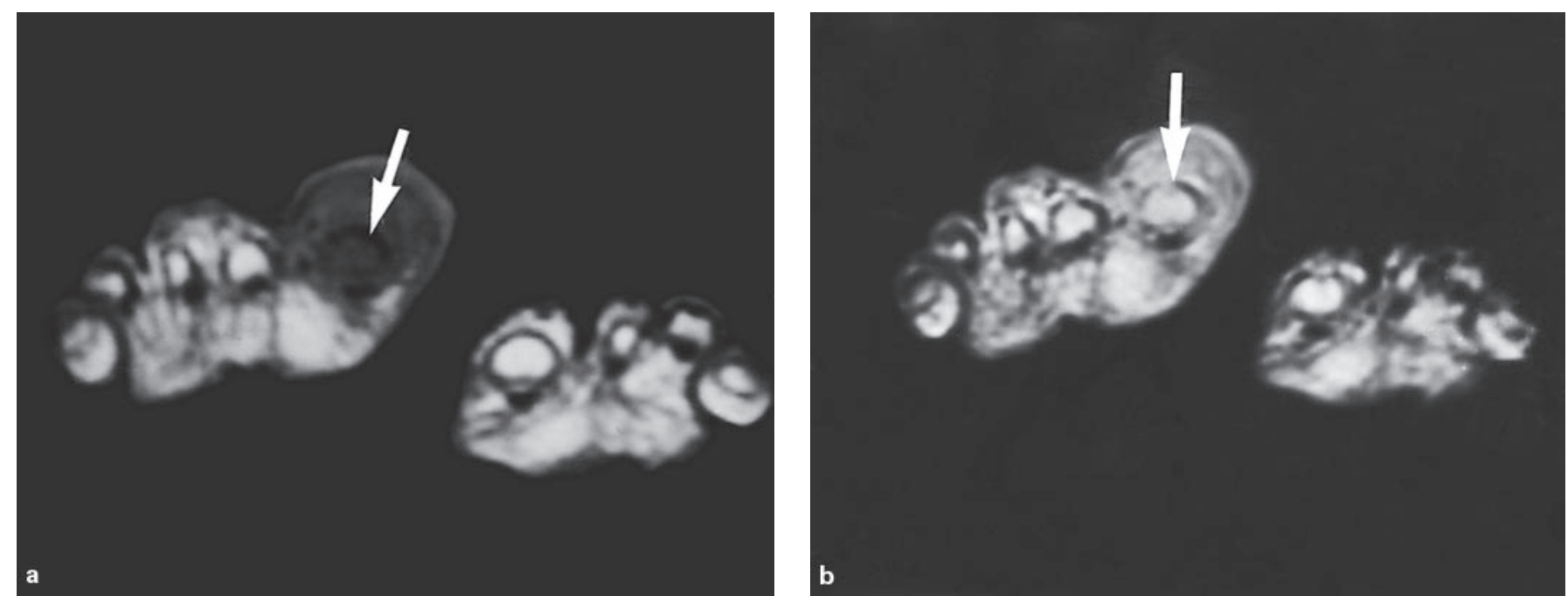

Fig. 2. Osteomyelitis with coexisting cellulitis. a Transverse $T_{1} W I$ of the forefoot shows low signal intensity of the bone marrow of the right 1st toe distal phalanx (arrow). b Post gadolinium, focal bone marrow enhancement (arrow) and diffuse enhancement of overlying soft tissue is seen. Osteomyelitis with cellulitis was confirmed by subsequent tissue biopsy results.

(TR $4300 \mathrm{~ms}$, TE $80 \mathrm{~ms}$, NEX 3) were performed on the affected feet with slice thickness of $4 \mathrm{~mm}$ and spacing of $1 \mathrm{~mm}$. Matrix size was $256 \times 224$ and the total scan time was, on average, $40 \mathrm{~min}$. In 2 cases contrast was not given because the patients could not tolerate the scanning time. Two consultant radiologists qualified in MRI (H.K., O.S.) evaluated the MR images; the final MR diagnosis was made by consensus. The MRI findings were compared to subsequent postsurgical histopathological findings. In the cases treated conservatively, MR diagnosis was compared with clinical follow-up including laboratory findings, physical examination, clinical impression and eradication of symptomatology following antibiotic therapy. The images were evaluated for the presence of infection (osteomyelitis, deep seated abscess, and cellulitis), tenosynovitis, and/or neuropathic joint, and the site and extent of the pathology to bones and soft tissues.

\section{MRI Criteria}

MRI imaging criteria used for the diagnosis of osteomyelitis, abscess, cellulitis, tenosynovitis and neuropathic joint were based on those described in previous studies [4-9]. Osteomyelitis was diagnosed when focally increased bone marrow signal on $\mathrm{FST}_{2} \mathrm{WI}$ (fig. 1a) and focally decreased marrow signal on $\mathrm{T}_{1} \mathrm{WI}$ with or without cortical destruction, and focal marrow enhancement on postcontrast $\mathrm{T}_{1} \mathrm{WI}$ was observed (fig. 1,2). Normal marrow signal on $\mathrm{T}_{1} \mathrm{WI}$ with high signal on $\mathrm{FST}_{2} \mathrm{WI}$ and marrow enhancement post contrast were also considered as osteomyelitis as reported by other investigators $[9,10]$. Abscess was diagnosed when a well-defined high signal intensity collection was seen in the soft tissues on $\mathrm{FST}_{2} \mathrm{WI}$ with or without thick rim enhancement on post-contrast $\mathrm{T}_{1} \mathrm{~W}$ sequences [11]. Cellulitis was diagnosed when soft tissues exhibited high signal on $\mathrm{FST}_{2} \mathrm{WI}$ with diffuse enhancement on postcontrast images on $\mathrm{T}_{1} \mathrm{~W}$ in the absence of bone changes [11] (fig. 2, 3 ). Infected tenosynovitis was diagnosed when high signal intensity fluid was identified within tendon sheaths on $\mathrm{FST}_{2} \mathrm{WI}$ and it was close to an ulcer or other clinical evidence of infection, to differentiate it from mechanical tenosynovitis. The diagnosis of acute rapidly progressing neuropathic joint was made when there was low intensity marrow signal on $\mathrm{T}_{1} \mathrm{WI}$ and high marrow signal on $\mathrm{FST}_{2} \mathrm{WI}$ at the subarticular regions associated with diffuse soft tissue edema and swelling or juxta-articular edema, and on postcontrast images the joint capsule and juxta-articular marrow and soft tissues demonstrated enhancement (fig. 3) [11]. Preserved subcutaneous fat is a finding that helps differentiate it from septic arthritis. Chronic neuropathic joint was diagnosed when irregular destruction of the subchondral cortices of a joint accompanied by low signal intensity of the underlying trabecular bone on $\mathrm{T}_{1} \mathrm{WI}$ and $\mathrm{FST}_{2} \mathrm{WI}$ was identified $[9,11]$; edema and enhancement were absent or less prominent. In addition, there is usually joint disorganization, subluxation or deformity. The final MRI diagnosis was verified in all patients with review of microbiologic $(n=10)$ or histopathological reports $(n=19)$.

\section{Histological Criteria}

A positive diagnosis of osteomyelitis was established by means of culture growth or characteristic histological findings including aggregates of inflammatory cells (neutrophils, lymphocytes, histocytes and plasma cells), erosion of trabecular bone, and bone marrow changes that ranged from loss of normal marrow fat with acute osteomyelitis to fibrosis and reactive bone formation with chronic disease. Acute rapidly progressing neuropathy was diagnosed when degenerative osteoarthritis changes without inflammation were seen, and chronic neuropathy when there was evidence of osteoarthritic changes with surrounding bone production (including subchondral sclerosis and osteophytes, intra-articular bodies and debris and subchondral cysts) and bone erosion. Cellulitis was diagnosed when there were acute inflammatory cells in the subcutaneous connective tissues and, sometimes, suppuration. 

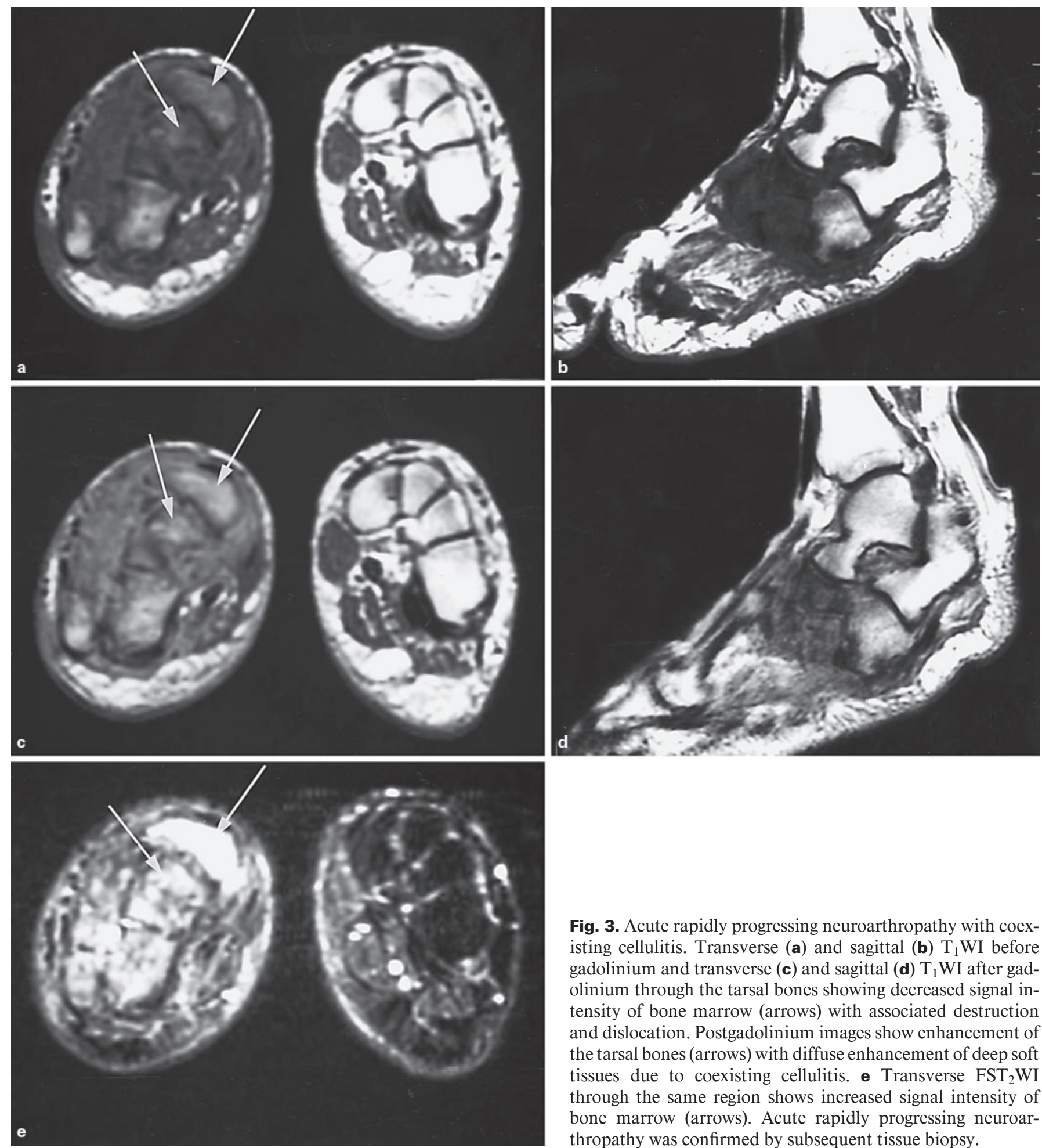

Fig. 3. Acute rapidly progressing neuroarthropathy with coexisting cellulitis. Transverse (a) and sagittal (b) $\mathrm{T}_{1} \mathrm{WI}$ before gadolinium and transverse (c) and sagittal (d) $\mathrm{T}_{1} \mathrm{WI}$ after gadolinium through the tarsal bones showing decreased signal intensity of bone marrow (arrows) with associated destruction and dislocation. Postgadolinium images show enhancement of the tarsal bones (arrows) with diffuse enhancement of deep soft tissues due to coexisting cellulitis. e Transverse FST 2 WI through the same region shows increased signal intensity of bone marrow (arrows). Acute rapidly progressing neuroarthropathy was confirmed by subsequent tissue biopsy. 
Table 1. Percent of correct MRI diagnoses based on histopathological $(n=19)$ and clinical $(n=10)$ follow-up diagnosis

\begin{tabular}{lcclcc}
\hline $\begin{array}{l}\text { Cases based } \\
\text { on MR diagnosis }\end{array}$ & $\begin{array}{l}\text { Cases with } \\
\text { histological } \\
\text { tissue sampling }\end{array}$ & $\begin{array}{l}\text { Cases with correct } \\
\text { final diagnosis } \\
\text { based on HP }\end{array}$ & $\begin{array}{l}\text { Cases with correct } \begin{array}{l}\text { \% of correct MR } \\
\text { final diagnosis } \\
\text { based on CFU }\end{array} \\
\begin{array}{l}\text { diagnosis based } \\
\text { on HP }\end{array}\end{array}$ & $\begin{array}{l}\text { \%orrect MR } \\
\text { diagnoses based } \\
\text { on CFU }\end{array}$ \\
\hline $\begin{array}{l}\text { Osteomyelitis, } \mathrm{n}=14 \\
\text { Neuropathy, } \mathrm{n}=8\end{array}$ & 14 & 11 & 0 & 79 & NA \\
Cellulitis, $\mathrm{n}=26$ & 5 & 5 & 3 & 100 & 100 \\
Normal, $\mathrm{n}=3$ & 19 & 19 & 10 & 100 & 100 \\
\hline
\end{tabular}

$\mathrm{HP}=$ Histopathology $\mathrm{CFU}=$ clinical follow-up; NA = not applicable.

\section{Treatment}

All patients were started on i.v. antibiotics (Tazocin) upon admission. Patients with wound or sinus had culture samples taken before starting antibiotics. The outcome was determined on the basis of operative findings or the clinical condition of patients continuing with conservative therapy. If the diagnosis was cellulitis, the antibiotic therapy was continued until the warmth, redness and edema subsided. If it was osteomyelitis, then appropriate antibiotics were used depending on the result of culture from an ulcer. If no ulcer was present, gentamycin, Flagyl and Ampiclox were given, and the antibiotics were continued for 6-7 weeks. If the patient underwent amputation then the antibiotics were continued for 1 week only.

\section{Results}

The MRI findings in 29 patients consisted of osteomyelitis in 14 patients, abscess in 5, tenosynovitis in 4, neuropathic joint in 8 , and cellulitis in 26 patients. Three cases were normal. Apart from the 3 normal cases, all the other 26 cases had coexisting cellulitis.

Of the 29 cases, 19 required surgical intervention. The procedures performed were amputation of third metatarsal bone in 1 patient, amputation of the lower end of the fibula in another, soft tissue debridement in 9, bone debridement in 5 , and soft tissue and bone debridement in 3 patients.

Of the 14 osteomyelitis cases diagnosed by MRI, the presence of infection was confirmed by pathologic examination of bone tissue in 11 cases (79\%) (table 1) but not in 3 cases $(21 \%)$. Of the 3 cases, 2 were negative for osteomyelitis on histology but positive for acute neuropathic joint. In the 3rd case the histological confirmation was not obtained and the patient healed after conservative therapy and was diagnosed as cellulitis on clinical followup. Of 29 patients 26 were diagnosed as having coexisting cellulitis. Nineteen of them were confirmed histologically and other 10 on clinical follow-up (table 1). Clinically, all patients were treated as cellulitis with eradication of symptomatology following antibiotic therapy.

Of 8 patients diagnosed on MRI as having neuropathic joint, 5 were confirmed histologically and 3 on clinical follow-up (table 1). All the 8 patients had infections in the form of cellulitis $(n=5)$ or both osteomyelitis and cellulitis $(n=3)$. Three cases had clinical manifestation of neuropathic joints but on MRI there were no features of neuropathy. Four cases diagnosed as tenosynovitis by MRI were not confirmed histologically, as these patients were treated conservatively.

In the cases in which histological tissue sampling (19 out of 29 cases) was present, MRI accurately depicted the diagnosis, site and extent of infection in 17 cases $(90 \%)$. In 2 cases $(0.7 \%)$ the MRI diagnosis was osteomyelitis, although histopathological diagnosis was consistent with acute rapidly progressing neuropathy. The remaining 10 cases did not undergo surgical treatment and therefore no histological proof of the diagnosis could be obtained, hence the final diagnosis was based on clinical followup.

Focal bone marrow enhancement (fig. 1, 2) was seen in 10 cases. In 7 of them (70\%) osteomyelitis was proved histologically and 2 cases had acute rapidly progressing neuropathy (fig. 3). One case did not have histological proof and the patient was healed conservatively; diagnosis on clinical follow-up was cellulitis. None of the other cases showed this pattern of bone marrow enhancement.

Our study showed that overall diagnostic accuracy of MRI in diabetic foot is high (84\%). The sensitivity and specificity of MRI in diagnosing osteomyelitis were 100 and $63 \%$, respectively. The positive and negative predictive values were 79 and $100 \%$, respectively. Table 2 shows a comparison of MRI and histopathology for diagnosis of osteomyelitis. 
Table 2. Comparison between MRI and histopathology in diagnosing osteomyelitis

\begin{tabular}{lclr}
\hline & $\begin{array}{l}\text { Osteomyelitis } \\
\text { by MRI }\end{array}$ & $\begin{array}{l}\text { No osteomyelitis Total } \\
\text { by MRI }\end{array}$ \\
\hline Osteomyelitis by histology & $11(\mathrm{TP})$ & $0(\mathrm{FN})$ & 11 \\
No osteomyelitis by histology & $3(\mathrm{FP})$ & $5(\mathrm{TN})$ & 8 \\
Total & 14 & 5 & 19 \\
\hline
\end{tabular}

$\mathrm{TP}=$ True positive $; \mathrm{FP}=$ false positive $; \mathrm{FN}=$ false negative $;$ $\mathrm{TN}=$ true negative.

The extent of infection was evaluated to determine whether it involved the bones, the deep and superficial soft tissues and the joints, and for the presence of abscess and pockets of infections. MRI helped surgical planning for limb salvage procedures in 6 of the patients with osteomyelitis and in 1 case which was clinically suspected to have osteomyelitis and proved to have cellulitis on MRI and histopathology. Follow-up studies confirmed that all patients with complete resection of infected bones healed completely.

\section{Discussion}

Osteomyelitis of diabetic patient foot is very common especially in the presence of angiopathy. It occurs in 15\% of diabetics [12] and needs prompt, early and accurate diagnosis and management to avoid amputation. Early diagnosis both clinically and radiologically is often difficult and is a challenge to both the clinician and the radiologist because of the common presence of superimposed disease processes such as neuroarthropathy, chronic soft tissue infection and edema. It is difficult to differentiate it from neuroarthropathy, which is another common disorder of diabetic foot, occurring in approximately 30 $60 \%$ of patients [13]. Neuroarthropathy has better prognosis and does not require amputation as a treatment option [3]. The treatment of early osteomyelitis consist of i.v. antibiotics for at least 4-6 weeks, whereas soft tissue infection needs only 1 week treatment and neuroarthropathy does not require antibiotic treatment [3]. Noninfected neuropathic ulcers and neuropathic joints can be managed conservatively. Ischemic limb can be managed medically in approximately $70 \%$ of cases, the reminder requiring revascularization.

The commonest imaging modality for the diagnosis of osteomyelitis is conventional radiography, which shows the abnormalities only after 10-21 days of the onset of infection when there is $30-50 \%$ bone destruction, seen as decreased bone density [14]. The radiological findings are not reliable in establishing the diagnosis in patients with violated bone which will give nonspecific findings, being diagnostic in only $3-5 \%$ of culture-positive cases. Conventional radiographs have proved to be neither sensitive nor specific for the diagnosis of osteomyelitis $[13,15$, $16]$.

The other imaging modality is multiphase ${ }^{99 \mathrm{~m}} \mathrm{Tc}-\mathrm{MDP}$ bone scan. This technique was considered as the imaging modality of choice for diagnosing osteomyelitis, becoming positive within 24-48 h of the onset of symptoms [17]. Bone scan is very sensitive but not specific for detection of infection in diabetic patients as it is also positive in diabetic neuroarthropathy [16]. Therefore, three-phase bone scan cannot reliably separate infection from rapidly progressive neuroarthropathy [18]. Attempts to improve the specificity of the three-phase bone scan were reported by Seldin et al. [19], in which they evaluated the arterial hyperemia, with a reported sensitivity of $94 \%$ but a specificity of $79 \%$. A drawback of the three-phase bone scan is that it takes $4 \mathrm{~h}$ to complete the study and sometimes a fourth phase is needed after $24 \mathrm{~h}$. Moreover bone scan remains positive for a long time, which is not helpful as a follow-up modality to assess the response of treatment.

Gallium-67 citrate scan is not helpful in resolving the question of osteomyelitis of the foot in diabetic patients, because it is also positive in noninfected neuroarthropathy [20]. Indium-111 leukocyte imaging has been reported to be both sensitive and specific for diabetic foot infections [21]. However, its sensitivity ranges from 50 to $100 \%$ and specificities from 29 to $100 \%$ [15, 21]. False positive results have been reported in several conditions, including rapidly progressive neuroarthropathy. Due to the poor spatial resolution in indium-111 leukocyte studies, uptake in soft tissues could be incorrectly attributed to bone uptake and vice versa $[15,21]$. Bone imaging combined with labeled leukocyte imaging improves the diagnostic accuracy of foot osteomyelitis and its differentiation from the soft-tissue infection. Crerand et al. [22] reported a sensitivity of $93 \%$ and a specificity of $83 \%$ for this dual isotope technique.

MRI has an excellent advantage in its ability to clearly differentiate soft tissue from bone infection due to its excellent spatial resolution, it is noninvasive, no radiation or radioactive materials are needed, and has the multiplanar capability [14]. Several investigators [14, 23] found MRI to be clearly superior to conventional radiog- 
raphy and bone scintigraphy, with a sensitivity and specificity approaching 100\% [4-9, 24], although some patients with acute fractures, recent surgery and acute neuropathic arthropathy can have juxta-articular marrow signals that mimic those of osteomyelitis $[11,25]$. In the present study, the sensitivity and specificity of MRI in diagnosing osteomyelitis were 100 and $63 \%$, respectively, which is comparable to previously reported studies [6, 24]. One of the significant observations in our series was that the MRI findings of chronic neuropathic joint (low $\mathrm{T}_{1}$, low $\mathrm{T}_{2}$ ) were consistently different from those of osteomyelitis (low $\mathrm{T}_{1}$, high $\mathrm{T}_{2}$ ) including the pattern of bone marrow enhancement on postgadolinium studies. Differentiation of osteomyelitis and acute neuropathic osteoarthropathy can be difficult since both can demonstrate marrow edema and enhancement, joint effusion and surrounding soft tissue edema $[11,26]$. There are however certain other findings that help in the diagnosis of osteomyelitis $[4,6,8]$. These include the presence of ulcer adjacent to the involved bone, presence of cellulitis and sinus tract, predominant involvement of one bone, typical location in the forefoot, mainly toe tips, metatarsal heads especially 1 st and 5th (fig. 1), calcaneus and malleoli, whereas acute neuropathy usually affects multiple bones (fig. 3) and joints at mid-foot (metatarsophalangeal joints, Lisfranc's joint and Chopart's joint) accompanied by usually intact skin and subcutaneous tissue $[8,11]$. The distinction between these two entities is important and proved to be clinically useful in our group of patients allowing conservative symptomatic therapy in the noninfected group.

Gadolinium helps in the diagnosis of osteomyelitis and was shown to have $89 \%$ sensitivity and $100 \%$ specificity in a study by Croll et al. [24], which is similar to our study. However, enhancement with gadolinium was also seen in 2 cases of acute neuropathy which we misdiagnosed on MRI, akin to reports by other investigators [25, 26]. In our study, focal marrow enhancement with contrast was seen in 70\% (7 out of 10) of histologically proved osteomyelitis cases and in 3 cases which were not proved histologically. In 2 of these patients acute neuroarthropathy was present, which could explain the marrow enhancement giving the false positive MRI diagnosis. The rest of the cases did not show marrow enhancement. Therefore, we agree with previously reports that bone marrow with normal signal intensity and absence of enhancement excludes the diagnosis of osteomyelitis [27]. In all patients with cellulitis, there was enhancement of superficial and/or deep soft tissues.
MRI has the ability to image bone marrow and provide excellent anatomical detail for planning of surgical procedures, particularly where a balance between resection of all infected material and preservation of normal bone is necessary for optimal function, even if the diagnosis of osteomyelitis was made by conventional radiography or scintigraphy [28]. In the present study, 1 of the patients had a limb salvage procedure because MRI was negative for osteomyelitis. Three of our patients failed to settle satisfactorily, despite having sepsis or necrosis dealt with surgically. Their repeated MRI showed pockets of infection not found at operation, similar to observation reported by Durham et al. [29]. Negative MRI gave the clinician confidence to continue conservative treatment [30]. Overall, in the cases in which we had histological correlation (19 out of 29 cases), MRI accurately depicted the diagnosis and the site and extent of infection in 17 cases (90\%), similar to findings reported by Lederman et al. [6].

One of the limitations of our study was the relatively long scanning time, especially in the older patients who could not keep their feet still for such a period, resulting in movement artifacts. In some of these patients we were unable to do a gadolinium study. All patients were scanned using a head coil, since we did not have a dedicated musculoskeletal coil, and both feet were tied together inside the coil to reduce movement artifact and for better comfort of the patient. The transverse images were done for both feet for comparison purposes, resulting in inevitable reduction in resolution and signal-to-noise ratio, which was another significant limitation in the study. Moreover, fat-suppressed $\mathrm{T}_{1} \mathrm{~W}$ sequences with and without contrast would give more information of abnormalities in bone marrow, but in our scanner the fat-suppressed images were of poor spatial resolution so we stopped doing them.

\section{Conclusion}

The results indicate that MRI is a sensitive and accurate imaging modality for the evaluation of foot infections in diabetic patients and for planning proper treatment. 


\section{References}

1 Abdella N, Al-Arouj M, Al-Nakhi, Al-Assousi A, Moussa M: Non-insulin-dependent diabetes in Kuwait: Prevalence rates and associated risk factors. Diabetes Res Clin Pract 1998;42:187_ 196.

-2 Humphrey LL, Palunbo PJ, Butters MA, Hallett JW Jr, Chu CP, O'Fallon WM, Ballard DJ: The contribution of non-insulin-dependant diabetes to lower extremity amputation in the community. Arch Intern Med 1994;154:885892.

3 Sinalore DR, Withrinton NC: Recognition and management of neuropathic (Charcot) arthropathies of the feet and ankle. J Hosp Sports Phys Ther 1999;29:736-746.

-4 Ledermann HP, Morrison WB, Schweitzer ME: Pedal abscesses in patients suspected of having pedal osteomyelitis: Analysis with MR imaging. Radiology 2002;224:649-655.

5 Ledermann HP, Morrison WB, Schweitzer ME, Raikin SM: Tendon involvement in pedal infection: MR analysis of frequency, distribution and spread of infection. AJR Am J Roentgenol 2002;179:939-947.

-6 Ledermann HP, Morrison WB, Schweitzer ME: MR image analysis of pedal osteomyelitis: Distribution, patterns of spread and frequency of associated ulceration and septic arthritis. Radiology 2002;223:747-755.

7 Morrison WB, Ledermann HP, Scheitzer ME: MR imaging of the diabetic foot. Magn Reson Imaging Clin N Am 2001;9:603-613.

-8 Morrison WB, Ledermann HP: Work-up of the diabetic foot. Radiol Clin North Am 2002;40: 1171-1192.

9 Morrison WB, Schweitzer ME, Batte WG, Radrack DP, Russel KM: Osteomyelitis of the foot: Relative importance of primary and secondary MR imaging signs. Radiology 1998; 207:625-632.

10 Craig JG, Amin MB, Wu K, Eyler WR, van Holsbeeck MT, Antonio Bouffard J, Shirazi K: Osteomyelitis of the diabetic foot: MR imaging-pathologic correlation. Radiology 1997; 203:849-855.
11 Moore TE, Yuh WTC, Kathol MH, El-Khoury GY, Corson JD: Abnormalities of the foot in the patients with diabetes mellitus: Findings on MR imaging. AJR Am J Roentgenol 1991; 157:813-816.

2 Ramsey SD, Newton K, Blough D, McCulloh DK, Sandhu N, Reiber GE, Wagner EH: Incidence, outcome, and cost of foot ulcers in patients with diabetes. Diabetes Care 1999;22: 332-387.

13 Fedele D, Comi G, Coscelli C, Cucinotta D, Feldman EL, Ghirlanda G, Greene DA, Negrin P, Santeusanio F: A multicenter study on the prevalence of diabetic neuropathy in Italy. Diabetes Care 1997;20:838-843.

14 Bonakar- Pour A, Gaines VD: The radiology of osteomyelitis. Orthop Clin North Am 1983; 14:21-37.

15 Newman LG. Waller J, Palestro CJ, Hermann G, Klein MJ, Schwatrz M, Harrington E, Harrington M, Ronan SH, Stagnaro-Green A: Leukocyte scanning with In-11 is superior to magnetic resonance imaging in diagnosis of clinically unsuspected osteomyelitis in diabetic foot ulcers. Diabetes Care 1992;15:15271530.

16 Larcus G, Brown ML, Sutton RT: Diagnosis of osteomyelitis of the foot in diabetic patients: Value of In-111 leukocyte scintigraphy. AJR Am J Roentgenol 1991;157:527-531.

17 Handmaker H, Leonards R: The bone scan in inflammatory osseous disease. Semin Nucl Med 1976;6:95-105.

18 Giurini JM, Chizan JS, Gibbons GW, Habershaw GM: Charcot's disease in diabetic patients: Correct diagnosis can prevent progressive deformity. Postgrad Med 1991;89: 163-169.

19 Seldin DW, Heinken JP, Feldman F, Aldersonet PO: Effect of soft tissue pathology on detection of pedal osteomyelitis in diabetics. $\mathrm{J}$ Nucl Med 1985;26:988-993.

20 Knight D, Gary HW, Bessent RG: Imaging for infection: Caution required with the Charcot joint. Eur J Nucl Med 1988;13:523-526.

- 21 Newman LG, Waller J, Palestro CJ, Schwantz M, Klein MJ, Hermann G, Harrington E, Harrington M, Roman SH, Stagnaro-Green A: Unsuspected osteomyelitis in diabetic feet ulcers: Diagnosis and monitoring by leukocyte scanning with indium In-111 oxyquinoline. JAMA 1991;226:1246-1251.
22 Crerand S, Dolan M, Laing P, Bird M, Smith ML, Klenerman L: Diagnosis of osteomyelitis in neuropathic foot ulcers. J Bone Joint Surg Br 1996; 78:51-55.

23 Weinstein D, Wang A, Richard C, Stewart CA, Motz HA: Evaluation of magnetic resonance imaging in the diagnosis of osteomyelitis in diabetic foot infections. Foot Ankle 1993;14: $18-22$.

24 Croll SD, Nicholas GG, Osborne MA, Wasser $\mathrm{TE}$, Jones S: Role of magnetic resonance imaging in the diagnosis of osteomyelitis in diabetic foot infections. J Vasc Surg 1996;24:266270 .

-25 Seabold JE, Flickinger FW, Kao SCS, Gleason TJ, Kahn D, Nepora JV, Morsh JL: Indium111-leukocyte/technetium-99m-MDP bone and magnetic resonance imaging: Difficulty of diagnosing osteomyelitis in patients with neuropathic osteoarthropathy. J Nucl Med 1990; 31:549-556.

-26 Marcus CD, Ladam-Marcus VJ, Leone J, Malgrange $\mathrm{D}$, Bonnet-Gausserand FM, Menanteau BP: MR imaging of osteomyelitis and neuropathic osteoarthropathy in the feet of diabetics. Radiographics 1996;16:1337-1348.

27 Unger E, Moldfsky P, Gatenby R, Hostz W, Broder G: Diagnosis of osteomyelitis by MR imaging. AJR Am J Roentgenol 1988;150: 605-610.

28 Wang A, Weinstein D, Greenfield L, Chiu L, Chambers R, Stewart C, Hung G, Diaz F, Ellis T: MRI and diabetic foot infections. Magn Reson Imaging 1990;8:805-809.

29 Durham JR, Lukens ML, Camanini DS, Wright JG, Smead WL: Impact of magnetic resonance imaging on the management of diabetic foot infections. Am J Surg 1991;162:150154.

30 Cook TA, Rahim N, Simpson HCR, Galland RB: Magnetic resonance imaging in the management of diabetic foot infection. Br J Surg 1996;83:245-248. 